\title{
A Novel Framework for Tracking In-vitro Cells in Time-lapse Phase Contrast Data
}

\author{
Ketheesan Thirusittampalam \\ kethesan@eeng.dcu.ie \\ M. Julius Hossain \\ julius@eeng.dcu.ie
}

Ovidiu Ghita

ghitao@eeng.dcu.ie

Paul F. Whelan

paul.whelan@dcu.ie

\author{
Centre for Image Processing and \\ Analysis \\ Dublin City University \\ Dublin, Ireland \\ www.cipa.dcu.ie
}

\begin{abstract}
With the proliferation of modern microscopy imaging technologies the amount of data that has to be analysed by biologists is constantly increasing and as a result the development of automatic approaches that are able to track cellular structures in timelapse images has become an important field of research. The aim of this paper is to detail the development of a novel tracking framework that is designed to extract the cell motility indicators in phase-contrast image sequences. To address issues that are caused by nonstructured (random) motion and cellular agglomeration, cell tracking is formulated as a sequential process where the inter-frame cell association is achieved by assessing the variation in the local structures contained in consecutive frames of the image sequence. We have evaluated the proposed algorithm on dense phase contrast cellular data and the reported results indicate that the developed algorithm is able to accurately track MadinDarby Canine Kidney (MDCK) Epithelial Cells in image data that is characterised by low contrast and high level of noise.
\end{abstract}

\section{Introduction}

The precise estimation of cell motility allows the extraction of quantitative indicators that describe a wide range of biological processes including embryogenesis, inflammation, tumour development, etc. [ $\square, \mathbb{W}$ ]. This area of research has received substantial interest from cell and molecular scientists since the understanding of mechanisms that stimulate/reduce the cell motility has important practical implications []]. Typically, the cell motility is evaluated in sequences of time-lapse images and the current practice involves a manual procedure to determine the cell association in consecutive frames. However, with the advent of modern microscopy imaging modalities the amount of information required to be analysed by biologists has substantially increased and in many situations the manual cell tracking procedure has become impractical [四]. Thus, computer-based automatic techniques that are able to robustly track the cells in large image sequences have become an invaluable research tool when the tracking process is evaluated in serial investigations. As indicated above, cellular 
tracking has become an active area of research and many approaches have been proposed to locate, segment and track various types of cells in time-lapse data. Since the tracking process is often defined based on the characteristics of the cells present in the image, the proposed techniques were generally based on application dependent architectures. When these approaches are evaluated from a computer vision standpoint, two main categories can be identified. The first category contains segmentation-driven approaches where cells in each frame are detected first and then the detected cells are associated by optimising an objective function to enforce the continuity of the tracking process in the spatio-temporal domain $[\square, \mathbf{\square}, \mathbf{\theta}, \boldsymbol{\square}, \mathbb{\square}]$. These approaches have shown promising results when applied to sparse cellular data characterised by strong image contrast (fluorescence microscopy data) but they proved to be less successful when applied to low contrast data that exhibit cellular agglomeration, proliferation and image noise. The main weakness of these approaches resides in the rigid architecture that cannot adapt to errors that occur during the segmentation process, thus their extension to cell tracking in phase contrast images is not straightforward. The reliance of the tracking algorithms on the accuracy of the segmentation process forms one of the issues that will be addressed in this paper. Considering the fact that cells are difficult to be extracted in each frame, substantial research efforts have been devoted to develop model driven techniques where intensity attributes are parameterised and the tracking process is performed by evolving the data-driven model from frame to frame. Tracking al-

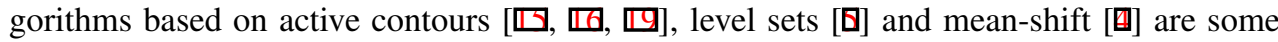
representative approaches included in this category. While model based approaches have associated the advantage that they do not require the segmentation of the cells in each frame, these methods are also problematic when applied to complex data, a situation when they are likely to be trapped in local minima. In addition, active contour and mean-shift-based algorithms do not naturally handle topology changes when tracking cells that exhibit random motion patterns and they have shown substantial limitations when dealing with issues caused by cellular agglomeration and proliferation. Consequently, Kalman and particle based filters were integrated within the standard model-based tracking schemes with a view of improving their performance $[\because, \square, \square]$, but the simple inclusion of the motion estimators within the tracking process proved also troublesome as they were not able to sample the modes of variation of the random cell motility. Thus, the research moved into the estimation of the motion patterns using multiple statistical models and algorithms that followed this approach have been reported in $[\boldsymbol{Q}, \square]$. Although these algorithms include multiple models corresponding to different cellular movement types, they entail a considerable level of supervision to train the data and motility models. As a result, recent approaches started to investigate the feasibility of hybrid procedures that investigate the cell tracking process from a more generic perspective and in this paper we propose a multi-step cell tracking strategy in which the neighbouring relationships of the cells that are detected during the segmentation process are encoded using a mesh representation based on Delaunay triangulation [ $[$ ] $]$. The neighbour relationship is utilised in a structural manner and in our approach structures of cells are matched rather than individual cells to adapt to tracking scenarios that are characterised by random motion. To compensate for segmentation errors we have developed a novel framework that first evaluates the local distortions in the Delaunay meshes in consecutive frames and then identifies the location of the missed cells using a pattern matching strategy. We have applied the proposed cell tracking algorithm on large phase-contrast image sequences and the experimental results indicate the efficiency of the proposed cell tracking framework in redressing the errors that occur during the segmentation process. 


\section{Overview of the cell-tracking algorithm}

The proposed cell tracking algorithm is constructed in a multi-step modular fashion. The first step of our algorithm addresses the cell segmentation and the proposed approach involves a completely automatic data driven procedure. The second component of the algorithm implements the tracking process where the cell association is based on the evaluation of the local similarity in the Delaunay mesh structures in consecutive frames of the image sequence. While the data returned by the segmentation algorithm is directly used in the process required to generate the Dealunay mesh in each frame, the segmentation errors that are caused by the improper contrast in image data will induce substantial errors during the tracking process.

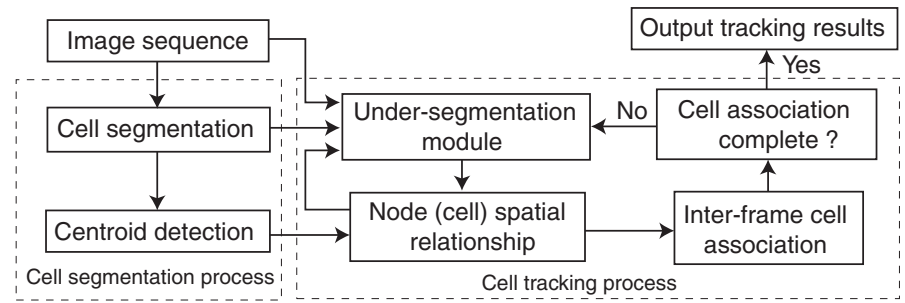

Figure 1: Overview of the proposed cell tracking framework.

To find a solution to this problem we have devised a multi-step tracking technique. In the first phase the proposed algorithm identifies the corresponding cells by evaluating the local similarities in the cellular structures, while in the second phase the algorithm attempts to reduce the level of unmatched nodes in the frames under investigation. The occurrence of unmatched nodes can be caused by several possibilities, including cellular agglomeration, under segmentation and the migration of the cells outside the region of interest that is imaged by the microscope. If such situations are encountered during the cell association process, the algorithm initiates a process that combines the information associated with the nodes that are matched in the first phase of the tracking process with the information in the intensity domain to redress the problems caused by segmentation errors. The block diagram that illustrates the logical connections between all modules of the tracking algorithm is shown in Figure 1.

\section{Cell segmentation module}

The aim of this module is to segment the cells in each frame of the image sequence and to identify their centroid points that are required to construct the Delaunay mesh. Since the MDCK phase contrast image data can be roughly approximated with a bi-modal distribution, the cells can be theoretically extracted using threshold-based segmentation techniques. However, purely threshold-based approaches are not a valid option as the contrast between the cells and background is variable within the same image and in addition the distribution of the intensity values within areas defined by cells is not constant. Moreover, substantial intensity offsets can occur when the cell data is analysed in different frames of the sequence, and this inserts an additional complication that has to be accommodated by the cell segmentation algorithm. Based on the problems mentioned above we have devised a cell segmentation scheme which attempts to maximally exploit the contrast difference between the cells and the background information. An overview of the proposed algorithm is depicted in Figure 2.

As Figure 2 illustrates, the proposed cell segmentation algorithm consists of several steps that are applied to identify the areas in the image that are characterised by relative large 
variations in the intensity domain.

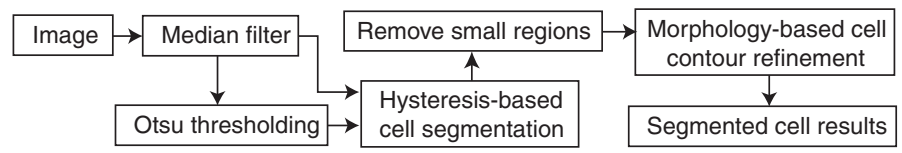

Figure 2: Outline of the cell segmentation algorithm.

As a result the initial cell seeds are extracted using the Otsu thresholding algorithm [प] that assumes that the intensity distribution of the data is bi-modal. However, since the distribution of the intensity values within the cell region is not constant, the data returned by the Otsu thresholding operation is characterised by errors such as holes and poor localization of the cell contours. To compensate for these errors, a hysteresis-based morphological operation is applied to grow the initial seeds extracted by the Otsu thresholding technique. Other components of the algorithm detailed in Figure 2 perform the impulse noise removal and the removal of small regions that are caused by dead cells and the debris present in the background medium where the cells are grown.

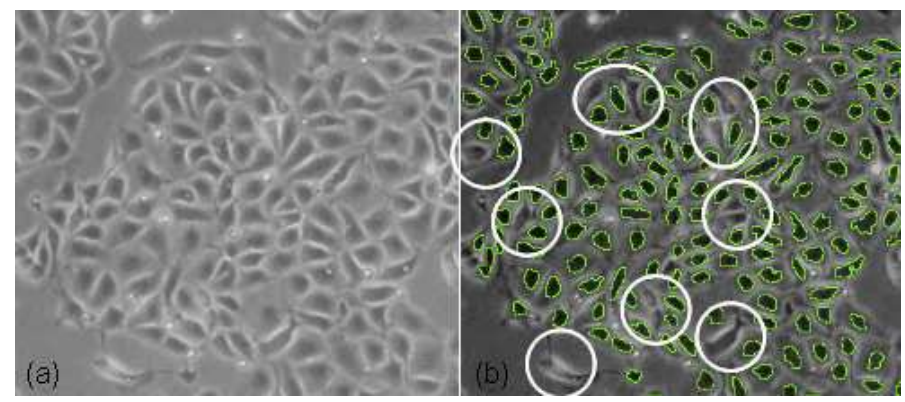

Figure 3: Cell segmentation process. (a) MDCK image. (b) Segmentation results. The cells that are not identified by the algorithm are highlighted with circular markers.

An example that illustrates the performance of the developed algorithm is shown in Figure 3, where it can be noticed that the algorithm fails to identify a number of cells due to the low contrast between the cell data and the background. Nonetheless, these errors will reduce the performance of the subsequent cell tracking process and as illustrated in Figure 1 we have devised a module that identifies the situations when under-segmentation occurs. This will be explained in the next section of the paper.

\section{Cell tracking module}

\subsection{Delaunay mesh generation}

After the cell segmentation routine is completed, the algorithm extracts the centroid points for all identified cells in the image sequence. It is useful to note that there are situations when cells are merged into clusters and to allow the identification of each individual cell the algorithm selects the centroid points using an approach that evaluates the peaks in the distance transformed data. For instance, if cells are merged in a cluster, this will generate multiple peaks in the distance transformed data and the algorithm divides the cluster into its 
constituent parts and assigns a centroid point for each cell in the cluster. In this way, each cell in the image sequence is represented by a point $C_{i}^{t}\left(x_{i}, y_{i}\right)$, where $i$ defines the index of the cell in the frame $t$. Once the centroid points are extracted, a Delaunay mesh is constructed for each frame in the image sequence as illustrated in Figure 4. In this fashion, the local structure in the Delaunay mesh for each cell (see the highlighted section in Figure 4b) provides a detailed description of the topological arrangement between the cell of interest and its closest neighbours and this information is used in the process of identifying the associated cells in adjacent frames of the sequence.
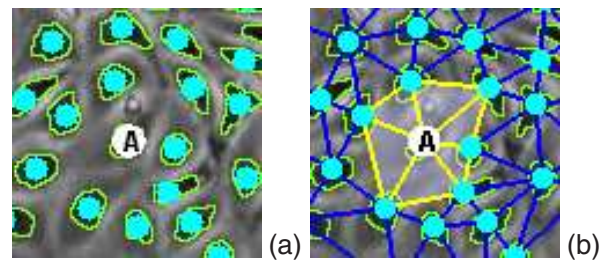

Figure 4: Delaunay mesh and local structure. (a) Cell centroid points. (b) The corresponding Delaunay mesh. The highlighted local structure defines the local relationship structure associated with the node labelled with $\mathrm{A}$ in figure (a)

\subsection{Cell association process}

The identification of the corresponding cells in adjacent frames is carried out by evaluating the level of similarity in the local structures of the Delaunay meshes that are calculated for the frames under investigation. As indicated in Section 3, one issue that needs to be accommodated by the cell association process is the occurrence of under-segmentation. In this situation, for cells that are identified in the previous frame $t$ we are not able to identify a corresponding cell in the current frame $t+1$. Nonetheless, the occurrence of undersegmentation significantly complicates the cell association process and to address this cell tracking scenario we have devised a three-step algorithm. In the first phase of the algorithm we attempt to identify the nodes (cells) that generate similar local structures in the Delaunay meshes calculated for the adjacent frames under analysis. The similarity between two nodes in the Delaunay meshes that correspond to the adjacent frames is evaluated as follows,

$$
M\left(u_{i}^{t}, u_{j}^{t+1}\right)=\frac{S\left(F_{i}^{t}, F_{j}^{t+1}\right)}{\left|F_{i}^{t}\right|}
$$

where $F_{i}^{t}$ and $F_{j}^{t+1}$ are the triangular structures that are associated with the nodes $u_{i}^{t}$ and $u_{j}^{t+1}$ in the two Delaunay meshes, $\left|F_{i}^{t}\right|$ denotes the number of triangles associated with the node $u_{i}^{t}$, and $S(\bullet)$ defines the number of triangles that are matched with respect to Hausdorff distance [ $[\mathbf{D}]$. As we mentioned earlier, in the first phase of the cell association process we attempt to match only the nodes in the frames $t$ and $t+1$ of the sequence for which we are able to find the most precise match with respect to a predefined threshold value for their local Delaunay meshes. In the second step of the cell association algorithm we attempt to match the un-associated nodes that are adjacent to the nodes that are identified in the first step (i.e. the nodes that are determined with the highest confidence level). In other words, in the second step we use the information that is similar in the Delaunay meshes to aid the identification of the corresponding nodes in the $t$ and $t+1$ frames whose local structure 
is changed by unstructured cell motility or degenerations that may occur in the Delaunay meshes. To accommodate changes in the local Delaunay meshes we evaluate the similarity between nodes using an expression that is derived from (1).

$$
M\left(u_{i}^{t}, u_{j}^{t+1}\right)=\frac{S\left(F_{i}^{t}, F_{j}^{t+1}\right)}{\left|F_{i}^{t}\right|}+\frac{R\left(E_{i}^{t}, E_{j}^{t+1}\right)}{\left|E_{i}^{t}\right|}+\left(1-\frac{D\left(c_{i}^{t}, c_{j}^{t+1}\right)}{D_{\max }}\right)
$$

where $R(\bullet)$ counts the number of most similar links between the nodes $u_{i}^{t}$ and $u_{j}^{t+1}$ with those that are identified in the first step (using Eq. 1), $E_{i}^{t}=\left\{e_{i, m}^{t}\right\}$ defines the links between the node $i$ with its connected reference nodes $m$ in the Delaunay mesh, $\left|E_{i}^{t}\right|$ denotes the number of links associated with the node $u_{i}^{t}, D(\bullet)$ is the $L_{2}$ distance between the centroid points $c_{i}^{t}$ and $c_{j}^{t+1}$ associated with the nodes $u_{i}^{t}$ and $u_{j}^{t+1}$, respectively, and $D_{\max }$ is a parameter that defines the maximum cell movement that can occur in adjacent frames. In (2) the last term can be thought of as a functional that penalises large movements between cells in frames $t$ and $t+1$.
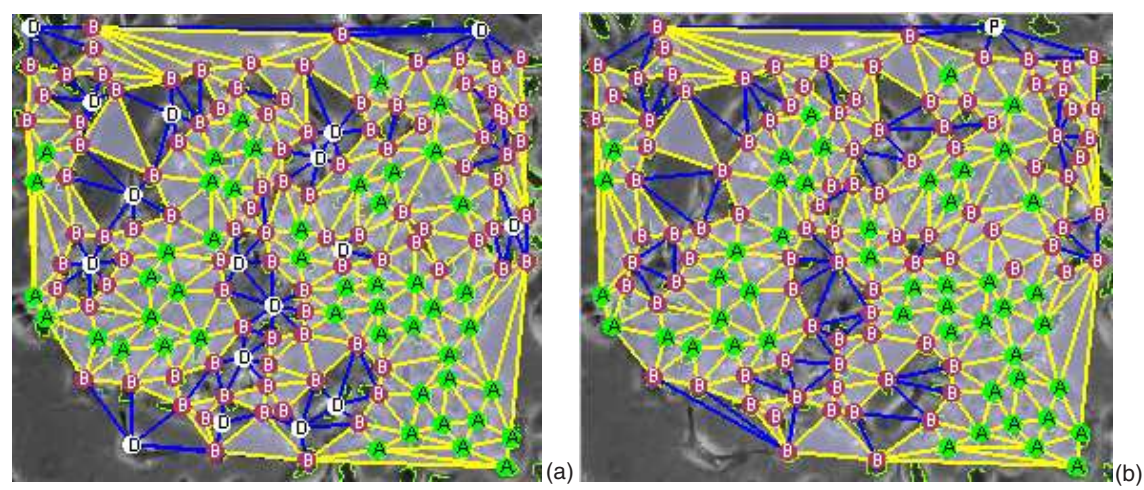

Figure 5: Illustration of the cell association process where the left and right images depict the $t$ and $t+1$ frames. (a) and (b) Cell association results - the nodes that are associated in the first and second steps are labelled with A and B, respectively. With D and P are marked the nodes in (a) and (b) that do not obey the matching criteria enforced with Eqns. (1) and (2). These nodes are not associated due to factors such as under-segmentation and large distortions in their local Delaunay meshes.

The cell matching process is illustrated in Figure 5 where the nodes that are associated in the first and second steps of the cell association algorithm are labelled with A and B, respectively. As Figure 5 illustrates, there are nodes that cannot be associated in the first two steps of the cell association process. These nodes were not associated due to inconsistencies in the local Delaunay meshes that were caused by issues related to under-segmentation, situations caused by nodes that enter or exit the region of interest (ROI) that is imaged by the microscope and cell division. However the nodes in frame $t+1$ that enter or exit the ROI and those that are the result of cell division were correctly left un-associated, as they do not have a corresponding node in the frame $t$. Based on this observation, in the third step of the cell association algorithm we attempt to redress the errors caused by under segmentation, a situation that occurs when nodes in the frame $t+1$ are not identified by the cell segmentation algorithm. 


\subsubsection{Redressing the problems caused by under-segmentation}

The occurrence of under-segmentation in frame $t+1$ leads to large distortions in the Delaunay meshes and as a consequence nodes in the frame $t$ are left un-associated after the application of the first two steps of the cell association algorithm. A typical scenario caused by under-segmentation is illustrated in Figure 6 which leads to the situation when the cell association algorithm is not able to find a corresponding cell in frame $t+1$ for the cell marked with a white dot in frame $t$ (see Figure 6a).

If cells are left un-associated in the frame $t$, the cell association algorithm activates the under - segmentation module in order to assess if a suitable location for a cell can be identified in frame $t+1$. To this end, we have developed an algorithm based on the Normalised Cross Correlation (NCC) [ $[\mathbf{\square}]$ that attempts to locate in the image $t+1$ a pattern that approximates the intensity profile of the rectangular template that encompasses the un-associated cell in frame $t$. To avoid the identification of potential cell locations that are far with respect to the position of the un-associated node in frame $t$, the search space in frame $t+1$ is restricted within a $D_{\max } \times D_{\max }$ area whose center is the coordinate of the centroid of the un-associated node. This process is shown in Figure 6 where the local maxima with respect to NCC are marked with white dots - Figure 6(c).
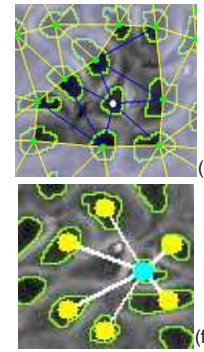
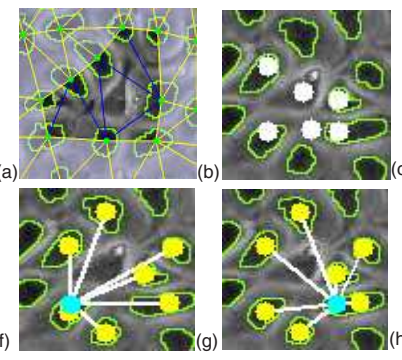
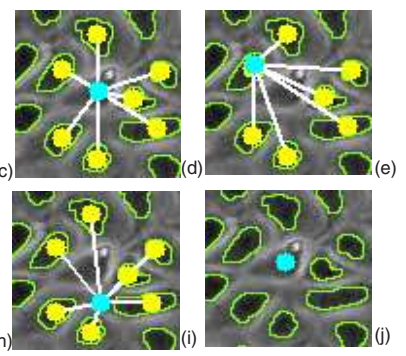

Figure 6: Best candidate selection. (a,b) Local distortions in the Delaunay meshes caused by under-segmentation. (c) The identified candidates cell locations for the missed cell in frame $t+1$ that is depicted in (b), (d-i) Evaluation of the mesh distortion for each candidate cell. (j) The candidate cell location that minimises the mesh distortion that is sampled by Eq. (3).

While within the $D_{\max } \times D_{\max }$ search area are several structures that resembles the intensity profile of the un-associated cell in frame $t$, the application of the normalised cross correlation procedure will generate multiple peaks. To identify the location that minimises the distortions in the local mesh in frame $t+1$ with respect to the local mesh in frame $t$, the distortion level is evaluated using the following expression,

$$
D\left(u^{t}, v^{t+1}\right)=\left(\sum_{j=1}^{m} \frac{\left|e_{u, j}^{t}-e_{v, j}^{t+1}\right|}{\operatorname{Max}\left(e_{u, j}^{t}, e_{v, j}^{t+1}\right)}+\sum_{j=1}^{m} \frac{\left|\theta_{u, j}^{t}-\theta_{v, j}^{t+1}\right|}{\operatorname{Max}\left(\theta_{u, j}^{t}, \theta_{v, j}^{t+1}\right)}\right)
$$

where $u^{t}$ is the un-associated node in frame $t, v^{t+1}$ is the candidate cell location in frame $t+1, m$ denotes the number of links between the node $u^{t}$ and the matched nodes identified in the first step of the cell association process, $e_{u, j}^{t}$ denotes the edge link between the node $u^{t}$ and the matched node with index $j$ in frame $t$ and $\theta_{u, j}^{t}$ defines the angle between the edge link generated by the node $u^{t}$ and the matched node with index $j$ with respect to the horizontal axis. Similarly, the same description applies to the notations used for the candidate cell 
location $v^{t+1}$. It is useful to note that for simplicity reasons the local mesh is created by connecting $u^{t}$ to each of the nodes that were identified in the first and second steps of the tracking process (see Eqns. 1 and 2). As indicated above the objective is to minimise the expression shown in Eq. (3) and this process is graphically illustrated in Figure 6(d-i). The tracking results when the third step of the cell association algorithm is applied to the $t$ and $t+1$ frames depicted in Figure 5 are shown in Figure 7.
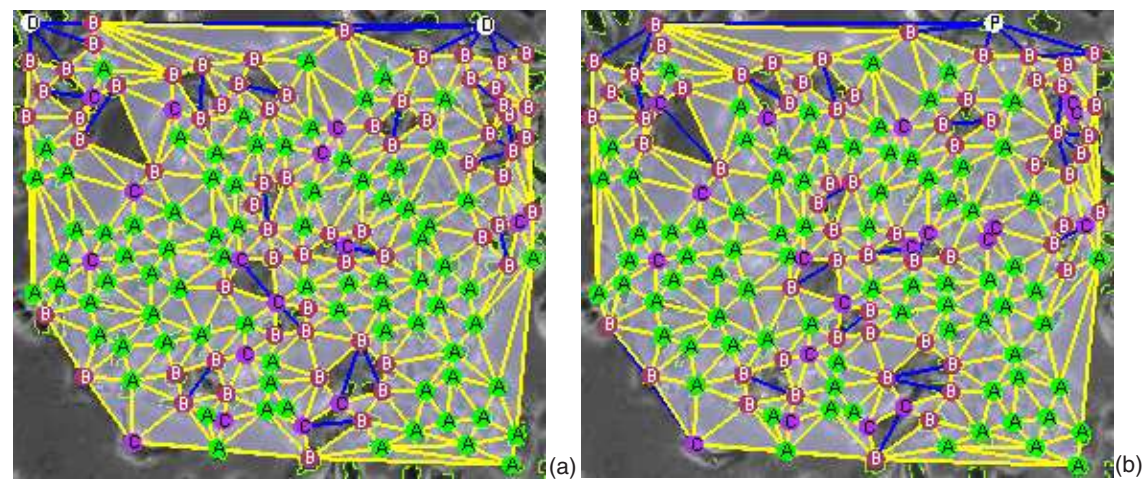

Figure 7: (a) and (b) Cell association results after redressing the problems caused by undersegmentation (the nodes associated in the third step of the cell tracking algorithm are labelled with C). Note that the algorithm excludes some marginally placed cells when the Delaunay meshes are generated as their detected centroid points lie within a certain distance threshold with respect to the borders of the image. This algorithmic choice is applied to restrict the occurrence of short tracks that are generated by cells that enter and exit the region of interest imaged by the microscope. These diagrams are best viewed in colour.

\section{Experimental results}

The proposed cell tracking algorithm was evaluated on MDCK Epithelial Cells. The spatial resolution of the image data is $400 \times 350$ and the temporal (inter-frame) resolution is 10 minutes. The results returned by the cell tracking algorithms are compared against the manually annotated data and the performance is evaluated using standard metrics such as the number of valid cells that are tracked in the overall image sequence. We have tested the algorithm on 4 MDCK image sequences and we were particularly interested to evaluate the efficiency of the proposed redressing scheme that is applied to counteract the tracking errors caused by under-segmentation. To assess the performance of the redressing scheme discussed in Section 4.2.1 we report experimental results in conditions when this procedure is removed (Delaunay Matching -DM) or included (Delaunay and Pattern Matching DPM) in the overall implementation of the proposed cell tracking algorithm. While undersegmentation induces distortions in the local neighbouring relationships (Delaunay meshes) that are used in the process of identifying the corresponding cells in adjacent frames, we first analyse the performance achieved by the segmentation procedure that is detailed in Section 3. When applied to the four MDCK sequences, the algorithm was able to achieve an overall detection accuracy of $94.23 \%$ ( 14440 cells where correctly identified out of a total of 15323 cells). The lowest accuracy was $90 \%$ and the highest was $97 \%$. As indicated earlier, the tracking accuracy is evaluated based on the number of cells that are correctly tracked in the 
entire image sequence and experimental results are reported in Table 1.

The experimental results reported in Table 1 clearly indicate that the inclusion of the component that is designed to address the under-segmentation in the development of the cell tracking algorithm substantially improve the accuracy of the cell tracking process. Graphical examples that sample the performance of the proposed cell tracking algorithm are shown in Figure 8 where it can be observed that the inclusion of the computational module that is designed to redress under-segmentation considerably reduces the occurrence of broken tracks.

\begin{tabular}{|c|c|l|c|c|c|c|}
\hline Cell sequence & \multirow{2}{*}{$\begin{array}{l}\text { Number of } \\
\text { frames }\end{array}$} & \multirow{2}{*}{$\begin{array}{l}\text { Number of cells } \\
\text { tracked throughout } \\
\text { the sequence(manually) }\end{array}$} & $\begin{array}{l}\text { Number of cells } \\
\text { tracked throughout } \\
\text { the sequence }\end{array}$ & Percentage & $\begin{array}{l}\text { Number of cells } \\
\text { tracked throughout } \\
\text { the sequence }\end{array}$ & Percentage \\
\hline 1 & 100 & 190 & 96 & $51 \%$ & 170 & $89 \%$ \\
\hline 2 & 100 & 120 & 59 & $49 \%$ & 105 & $88 \%$ \\
\hline 3 & 100 & 174 & 94 & $54 \%$ & 143 & $82 \%$ \\
\hline 4 & 100 & 135 & 58 & $43 \%$ & 112 & $83 \%$ \\
\hline
\end{tabular}

Table 1: Accuracy of the cell tracking algorithm when the module designed to redress the under-segmentation is removed (DM) or included (DPM) in the overall tracking framework.
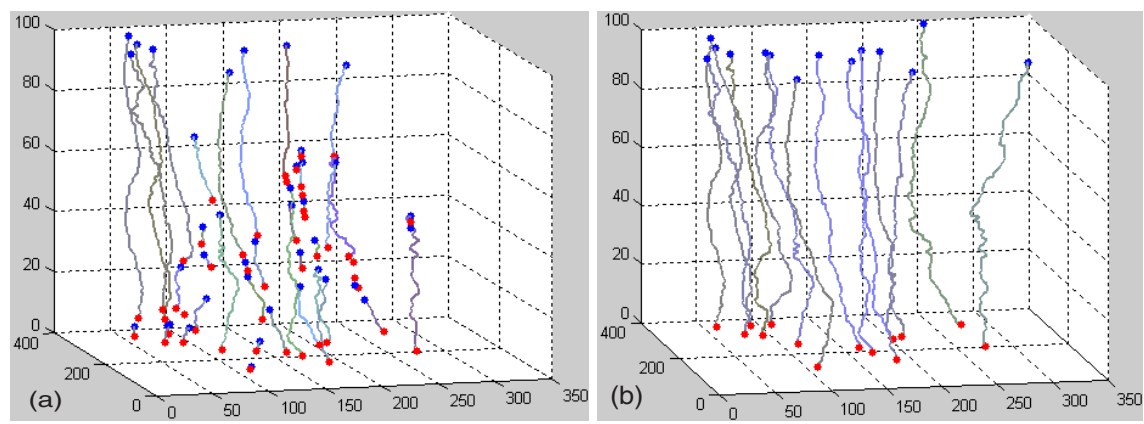

Figure 8: The effect of under-segmentation on the overall performance of the cell tracking process. (a) Cell tracking results - DM. (b) Cell tracking results - DPM.

\section{Conclusion}

In this paper we have detailed the development of a fully automatic cell tracking algorithm where the tracking process evaluates the similarities in the neighbouring relationships between cells in adjacent frames. In this paper we were particularly interested to evaluate the impact of the cell segmentation errors on the cell tracking accuracy and to robustly address this problem we have introduced a novel approach that redresses the under-segmentation problems using a pattern matching approach. We have applied the proposed strategy on large MDCK image sequences and the reported tracking accuracy is in the range 82 to $89 \%$. It is useful to point out that the resulting cell tracking strategy does not require any a priori information in regard to motion patterns and it is able to accommodate difficult situations such as unstructured cellular motility, cellular agglomeration, shape deformation, intensity variation and image noise. 


\section{Acknowledgements}

This research was supported by National Biophotonics and Imaging Platform (NBIP) Ireland funded under the Higher Education Authority PRTLI Cycle 4, co-funded by the Irish Government and the European Union - /Investing in your future/. We would like to thank Dr. András Czirók, Department of Anatomy and Cell Biology, University of Kansas Medical Centre for his valuable suggestions during the development stage of the cell tracking algorithm.

\section{References}

[1] I. Adanja, O. Debeir, V. Megalizzi, R. Kiss, N. Warzée, and C. Decaestecker. Automated tracking of unmarked cells migrating in three-dimensional matrices applied to anti-cancer drug screening. Expl. Cell Res., 316(2):181-193, 2010.

[2] O. Al-Kofahi, R. J. Radke, S. K. Goderie, Q. Shen, S. Temple, and B. Roysam. Automated cell linage construction: A rapid method to analysis clonal development established with murine neural progenitor cells. Cell Cycle, 5(3):327-335, 2006.

[3] M. de Berg, O. Cheong, M. van Kreveld, and M. Overmars. Computational geometry: Algorithms and applications. Springer, 2008.

[4] O. Debeir, P. Van Ham, R. Kiss, and C. Decaestecker. Tracking of migrating cells under phase-contrast video microscopy with combined mean-shift processes. IEEE T MI, 24 (6):697-711, 2005.

[5] A. Dufour, V. Shinin, S. Tajbakhsh, N. G. Aghion, J. C. Olivo-Marin, and C. Zimmer. Segmenting and tracking fluorescent cells in dynamic $3 \mathrm{~d}$ microscopy with coupled active surfaces. IEEE T IP, 14(9):1396-1410, 2005.

[6] A. Genovesio, T. Liedl, V. Emiliani, W. J. Parak, M. Coppey-Moisan, and J. C. OlivoMarin. Multiple particle tracking in $3 \mathrm{~d}+\mathrm{t}$ microscopy: Method and application to the tracking of endocytosed quantum dots. IEEE T IP, 15(5):1062-1070, 2006.

[7] D. P. Huttenlocher, G. A. Klanderman, and W. J. Rucklidge. Comparing images using hausdorff distance. IEEE T PAMI, 15(9):850-863, 1993.

[8] S. Jaeger, Q. Song, and S. Chen. Dynamik: A software environment for cell dynamics, motility, and information tracking, with an application to ras pathways. Bioinformatics, 25(18):2383-2388, 2009.

[9] T. Ketheesan, M. J. Hossain, O. Ghita, and P. F. Whelan. Cellular tracking in time-lapse phase contrast images. In Proc. IMVIP, pages 77-82, 2009.

[10] F. Li, X. Zhou, J. Ma, and S. T. C. Wong. Multiple nuclei tracking using integer programming for quantitative cancer cell cycle analysis. IEEE T MI, 29(1):96-105, 2010.

[11] K. Li, E. D. Miller, M. Chen, T. Kanade, L. E. Weiss, and P. G. Campbell. Cell population tracking and lineage construction with spatiotemporal context. Med. Image Anal., 12(5):546-566, 2008. 
[12] K. Miura. Tracking movement in cell biology. In Adv. Biochem. Engin./Biotechnol. Springer 95, pages 267-295, 2005.

[13] S. K. Nath, F. Bunyak, and K. Palaniappan. Robust tracking of migrating cells using four-color level set segmentation. LCNS, 4179:920-932, 2006.

[14] N. Otsu. A threshold selection method from gray-level histograms. IEEE T SMC, 9(1): 62-66, 1979.

[15] N. Ray, S. T. Acton, and K. Ley. Tracking leukocytes in-vivo with shape and size constrained active contours. IEEE T MI, 21(10):1222-1234, 2002.

[16] A. Sacan, H. Ferhatosmanoglu, and H. Coskun. Celltrack: An open-source software for cell tracking and motility analysis. Bioinformatics, 24(14):1647-1649, 2008.

[17] I. Smal, K. Draegestein, N. Galjart, W. Niessen, and E. Meijering. Particle filtering for multiple object tracking in dynamic fluorescence microscopy images: Application to microtubule growth analysis. IEEE T MI, 27(6):789-803, 2008.

[18] D. Tsai and C. Lin. Fast normalized cross correlation for defect detection. Patt. Rec. Lett., 24(15):2625-2631, 2003.

[19] C. Zimmer, E. Labruyere, V. Meas-Yedid, N. Guillen, and J. C. Olivo-Marin. Segmentation and tracking of migrating cells in videomicroscopy with parametric active contours: A tool for cell based drug testing. IEEE T MI, 21(10):1212-1221, 2002. 